\title{
1 Recellularization of bronchial extracellular matrix with primary bronchial smooth muscle
}

2

cells

4 Selma Ben Hamouda ${ }^{1 *}$, Amandine $\operatorname{Vargas}^{1}$, Roxane Boivin ${ }^{1}$, Maria Angelica Miglino ${ }^{2}$, Renata

5 Kelly da Palma ${ }^{3}$, Jean-Pierre Lavoie ${ }^{1 *}$.

6

7

$8{ }^{1}$ Department of Clinical Sciences, Faculty of Veterinary Medicine, University of Montreal, St-

9 Hyacinthe, Quebec, Canada

10

$11{ }^{2}$ School of Veterinary Medicine and Animal Sciences, University of Sao Paulo, São Paulo, Brazil

12

$13{ }^{3}$ Institute for Bioengineering of Catalonia, Barcelona, Spain

14

15

$16 *$ Corresponding authors:

17 Jean-pierre.lavoie@umontreal.ca (JPL)

$18 \quad$ Selma.ben.hamouda@,umontreal.ca $(\mathrm{SBH})$

19 


\section{Abstract}

21 Severe asthma is associated with an increased airway smooth muscle (ASM) mass and

22 an altered composition of the extracellular matrix (ECM). Studies have indicated that

23 ECM-ASM cell interactions contribute to this remodeling and its limited reversibility

24 with current therapy. Three-dimensional matrices allow the study of complex cellular

25 responses to different stimuli in an almost natural environment. Our goal was to obtain

26 acellular bronchial matrices and then develop a recellularization protocol with ASM cells.

27 We studied equine bronchi as horses spontaneously develop a human asthma-like disease.

28 The bronchi were decellularized using Triton/Sodium Deoxycholate. The obtained

29 scaffolds retained their anatomical and histological properties. Using

30 immunohistochemistry and a semi-quantitative score to compare native bronchi to

31 scaffolds revealed no significant variation for matrixial proteins. A DNA quantification

32 and electrophoresis indicated that most of DNA was $29.6 \mathrm{ng} / \mathrm{mg}$ of tissue \pm 5.6 with

33 remaining fragments of less than $100 \mathrm{bp}$. Primary ASM cells were seeded on the

34 scaffolds. Histological analysis after recellularization showed that ASM cells migrated

35 and proliferated primarily in the decellularized smooth muscle matrix, suggesting a

36 chemotactic effect of the scaffolds. This is the first report of primary ASM cells

37 preferentially repopulating the smooth muscle matrix layer in bronchial matrices. This

38 protocol is now being used to study the molecular interactions occurring between the

39 asthmatic ECMs and ASM to identify effectors of asthmatic bronchial remodeling.

40 Keywords: Decellularization, recellularization, airway smooth muscle cells, extracellular

41 matrix, asthma. 


\section{Introduction}

43 Asthma is a progressive and multi-component respiratory syndrome. Remodeling of the

44 airways in asthma is characterized by structural changes leading to a thickening of the

45 bronchial wall, airflow obstruction, and hyperreactivity of the airways [1]. In human

46 asthma, tissue remodeling is believed to be only partially reversible or even irreversible

47 following conventional treatments (corticosteroids / bronchodilators) even during

48 extended periods of remission [2]. While airway remodeling is considered a target for

49 asthma, little is known of the mechanisms involved in its development and reversibility.

50 This is due to ethical considerations related to the invasiveness when sampling the

51 airways, and technical limitations of the current imaging techniques.

52 As compared to single-layer culture, three-dimensional (3D) cell culture improves

53 different cellular parameters including viability, adhesion, proliferation, etc. A study

54 comparing the culture of skin fibroblasts on natural 3D matrices to their monolayer

55 culture on different substrates revealed significant variations. Cell adhesion was 5 to 6

56 times greater on 3D matrix than on single-layer culture, cell migration was also increased

57 and the acquisition of a morphology mimicking the in vivo appearance of the cells

58 occurred faster [3]. A study of human embryonic stem cell differentiation found that

59 cells were physiologically and morphologically more representative of the native cells

60 when cultured on a 3D matrix than when cultured on flasks [4]. This is also supported by

61 the finding that pulmonary fibroblasts response to tumor necrosis factor (TNF) $\alpha$ was

62 increased in 3D culture when compared to monolayer culture [5]. There are different

63 types of 3D culture including: 1) in suspension, 2) in gel scaffold or, 3) in natural or

64 synthetic fibrous scaffold [6, 7]. Culturing cells on 3D bases is more difficult and 
65 requires longer culture time than in monolayer, but the results obtained are believed to be

66 more representative of the natural environment [6]. Thus, in order to evaluate the

67 behavior of airway smooth muscle (ASM) cells in the asthmatic airways, we aimed to

68 decellularize an equine respiratory bronchus while maintaining its architecture and

69 protein composition to allow recellularization by bronchial smooth muscle cells. Equine

70 bronchi were studied as horses spontaneously develop an asthma-like condition that

71 shares clinical and remodeling features with human asthma [8]. Furthermore, it was

72 shown in this model that the quantity of ASM is increased, and only partially reversible

73 even after 1 year of inhaled corticosteroids [9, 10]. Results of the present study suggest

74 that the bronchial smooth muscle cells preferentially colonize the bronchial smooth

75 muscle extracellular matrix. These findings may allow investigating the interactions

76 between smooth muscle cells and the extracellular matrix in the asthmatic airways.

\section{Material and methods}

\section{Animals}

79 Archived lung tissues from four asthmatic and three healthy control horses (5 mares and

802 geldings) aged 10-12 years from a tissue bank (htttp://www.btre.com) were studied.

81 Four additional lungs were obtained from a slaughterhouse. The experimental protocol

82 was approved by the ethical committee of the University of Montreal number Rech-1578.

\section{Bronchi decellularization}

84 Bronchi from $2^{\text {nd }}$ to $4^{\text {th }}$ generation were dissected from the surrounding lung tissues 85 within 2 hours after euthanasia. The bronchi were then snap frozen in liquid nitrogen and 
86 kept at $-80^{\circ} \mathrm{C}$ until used. These bronchi were thaw and decellularized using a protocol

87 previously described [11] with minor modifications. Briefly, two consecutive cycles of

88 detergent (Triton 1X, Sodium deoxycholate and sodium chloride) and enzymatic (DNase)

89 treatments were followed by sterilization with paracetic acid-ethanol under continued

90 agitation to ensure the elimination of any immunogenic cellular material that may hinder

91 recellularization. Sections of bronchi were then paraffin-embedded for histology or snap

92 frozen for DNA and protein isolation.

\section{Airway smooth muscle cell isolation and culture}

94 Airway smooth muscle (ASM) cells were isolated from the same horses in the first hour

95 after the death, as previously described [12]. In brief, the ASM layer was collected from

96 the first bronchial bifurcation and then immersed in a digestion medium (Dulbeco's

97 Modified Eagle Medium / F12 nutrient mix (Thermofisher, Waltham, MA) with 0.125

$98 \mathrm{U} / \mathrm{ml}$ Collagenase H (Sigma Aldrich, St. Louis, MO), $1 \mathrm{mg} / \mathrm{ml}$ Trypsin inhibitor (Sigma

99 Aldrich, St. Louis, MO), $1 \mathrm{U} / \mathrm{ml}$ elastase (Worthington biochemical, Lakewood, NJ), 1\%

100 Penicillin-Streptomycin (Wisent Inc., Saint-Jean-Baptiste, QC) and 0.1\% Fungizone

101 (Fisher Scientific, Hampton, NH)).

102 Cells (ASM) were seeded into ventilated cell culture flasks at 300,000 cells $/ \mathrm{cm}^{2}$ in 103 DMEM/F12 medium supplemented with $0.0024 \mathrm{mg} / \mathrm{ml}$ adenine, $10 \%$ non104 decomplemented fetal bovine serum (FBS) (Wisent Inc., Saint-Jean-Baptiste, QC), 1\% 105 Penicillin-Streptomycin and $0.1 \%$ Fungizone and cultured at $37^{\circ} \mathrm{C}$ and $5 \% \mathrm{CO}_{2}$ for 48 106 hours. Media was then changed every 48 hours until confluence was reached. Cells were 107 frozen between the first and $4^{\text {th }}$ passage $(\mathrm{P})$ in liquid nitrogen until being used. 


\section{Smooth muscle cells characterization}

109 ASM cells were characterized by flow cytometry before recellularization, as previously

110 described [12]. Briefly, cells were stained for intracellular markers with anti- $\alpha$-SMA

111 (mouse IgG2a, Sigma Aldrich, St. Louis, MO, 1/250), anti-desmin (rabbit polyclonal

112 IgG, Abcam, Cambridge, UK, 1/200) and anti-SMMHC (rabbit IgG, Biomedial

113 technologies, Stoughton, MA, 1/300) antibodies for 1 hour. Cells were then washed 3

114 times and incubated for 30 minutes in the dark with fluorescent dye-conjugated anti-IgG

115 antibodies. Isotype-matched control antibodies (mouse IgG2a and rabbit IgG) were used

116 as negative control. All signals greater than those of the isotype-matched control

117 antibodies were considered positive, and degree of staining was evaluated as the mean

118 fluorescence intensity and mean percentage of positive cells. This characterization

119 showed simultaneous expression of $\alpha$-SMA (mean \pm SEM) for $90 \% \pm 8.6$ cells, SMMHC

120 for $71 \% \pm 16$ cells and desmin for $85 \pm 9.2$ cells.

\section{Assessment of decellularization efficiency}

122 Decellularized bronchi were stained and compared to native bronchi using the Russel

123 modification of Movat Pentachrome [13]. The protocol was modified as the exposure

124 time to ferric chloride and to alcoholic safran solution was changed to 1 and 5 minutes,

125 respectively. Images were obtained at 100 and 200 magnifications using Panoptiq

126 software (version 2) connected to a Prosilica GT camera (model: GT1920C) mounted on

127 a Leica DM4000 B microscope.

128 DNA was isolated from $10 \mathrm{mg}$ of frozen native and from freshly decellularized bronchi

129 using DNeasy blood and tissue $\mathrm{Kit}^{\circledR}$ (Invitrogen, Hilden, DE) as recommended by the 
130 manufacturer. DNA was then visualized on agarose gel. Quantification of double-

131 stranded DNA before and after decellularization was done using the Qubit DNA BR

132 Assay kit (Invitrogen, Carlsbad, CA) according to the manufacturer instructions. Proteins

133 were extracted using T-PER (Thermofisher, Waltham, MA) and quantified using Qubit

134 Protein Assay Kit (Invitrogen, Carlsbad, CA).

135 Immunohistochemical staining for collagen I, collagen IV and fibronectin was performed

136 on $10 \%$ formalin preserved native and decellularized bronchi. Tissues were incubated

137 overnight with primary antibodies (collagen I; rabbit anti-bovine IgG, Cederlane,

138 Burlington, ON, dilution 1:500, collagen IV; mouse anti-human IgG, Dako, Carpinteria,

139 CA, dilution 1:50, and fibronectin; unconjugated rabbit polyclonal antibody, Biorbyt, San

140 Francisco, CA, dilution 1:150). The biotinylated secondary antibodies were applied at the

141 same concentrations as the primary antibodies for 45 minutes. Vectastain ABC kit

142 (Biolynx, Brockville, ON) was applied before DAB revelation (Vector Laboratories,

143 Peterborough, UK) and a counterstain with Harry's hematoxylin. Negative controls were

144 also prepared. They were stained with rabbit or mouse IgG instead of the primary

145 antibodies to reveal potential unspecific staining. Using the negative controls as a

146 benchmark, a semi-quantitative score was established for the basement membrane,

147 smooth muscle, blood vessel and lamina propria labeling as follows: Grade 0: Absence of

148 staining, Grade 1: Presence of staining. From this score, an average was established to

149 compare the labeling difference between native and decellularized bronchi. 


\section{Bronchi recellularization protocol}

151 Decellularized bronchi were split in two and secured on a sterile support, then cut into

152 small pieces of a maximum of $1 \times 1 \mathrm{~cm}$ and rinsed in sterile PBS $1 \mathrm{X}$. Tissues were then

153 placed in a 24-well plate (Costar, Washington, D.C.) and recellularized with the ASM

154 cells between P4 and P7 at a concentration of 158,000 cells $/ \mathrm{cm}^{2}$. One and a half

155 milliliters of medium were added to the culture under the same condition described

156 above. After a 48-hour incubation, allowing primary adhesion, $1 \mathrm{ml}$ of the medium was

157 changed in each well. Then, the medium was changed every other day. Tissues were

158 maintained in culture, in the same well, between 48 hours and 41 days or transferred at 31

159 days to a 6-well plate (Celltreat, Pepperell, MA) for 10 more days. Samples were

160 collected at day 2, 7, 14, 21 and 31 without tissue transfer and at day 41 with and without

161 tissue transfer (Supplemental figure 1).

162 Assessment of recellularization efficiency

163 Tissues were fixed in $10 \%$ formalin, paraffin-embedded then sliced at $4.5 \mu \mathrm{m}$ thickness

164 and stained using Movat Pentachrome histological staining protocol. The qualitative

165 assessment of recellularization was based on a visual examination of the recellularized

166 tissue sections under the optical microscope at 100 and 200 magnifications using the

167 Panoptiq software, as previously described.

168 An immunofluorescence staining of 5 fresh-frozen bronchi recellularized at day 41 was

169 performed for $\alpha$-SMA. Tissues were incubated with the primary antibody for 2 hours at

$17037^{\circ} \mathrm{C}(\alpha-S M A$ anti-mouse IgG2a, 1:250, Sigma Aldrich, St-Louis, MO). The fluorescent

171 secondary antibody (Goat anti-mouse IgG, 1:1000, Invitrogen, Carlsbad, CA) was 
172 incubated for 1 hour at room temperature. An isotype control was used as a benchmark

173 for positive staining. The slides were analyzed under Olympus Fluoview FV1000

174 confocal unit attached to the inverted Olympus IX81 microscope (Olympus Canada,

175 Richmond Hill, ON, Canada) and compared to their replicate on a Movat Pentachrome

176 staining to confirm the histological results.

177 For scan electron microscopy, the recellularized bronchi were fixed in $2.5 \%$

178 glutaraldehyde, washed and post-fixed in 1\% aqueous osmium tetroxide solution, and

179 dehydrated in increasing series of alcohol (70\% to $100 \%)$. After dehydration, the samples

180 were dried on the LEICA EM CPD 300 Critical Point Apparatus, mounted on carbon tape

181 and gold-plated on the Emitech K550 Metalizing Apparatus, photo-documented on the

182 LEO 435VP Scanning Electron Microscope at the Advanced Diagnostic Center by Image

183 - CADI - Faculty of Veterinary Medicine and Animal Science - University of São Paulo.

\section{Statistical analyses}

185 The values are expressed as mean \pm standard error of the means (SEM). Values of DNA

186 and total protein quantification were analyzed by use of the paired student test (GraphPad

187 Prism 7). Immunohistochemistry scores were analyzed by an exact chi-square test to

188 compare the prevalence of positive staining against the status of the bronchi (native or

189 decellularized) using SAS v.9.3. Values of $\mathrm{P} \leq 0.05$ were considered significant. 
190 Results

\section{Decellularization efficiency assessment}

\section{Histological assessment}

193 Visual examination under the optical microscope at magnifications 100 and 200

194 confirmed the absence of cellular structures in decellularized matrices in comparison to

195 the native bronchi (Fig 1). The epithelial cell layer was totally removed. It also showed a

196 preserved bronchial architecture after the decellularization process with a maintenance of

197 the tissue organization and the contents in collagen and elastic fibers.

\section{DNA quantification and electrophoresis}

199 A decrease in DNA concentration was observed in decellularized bronchi. The mean 200 DNA concentration in the native bronchi was $2529 \mathrm{ng} / \mathrm{mg}$ of tissue \pm 72.7 whereas it was $20129.6 \mathrm{ng} / \mathrm{mg}$ of tissue $\pm 5.6(\mathrm{p}<0.0001$; Fig $2 \mathrm{a})$ after decellularization. Agarose gel 202 electrophoresis revealed that the remaining double-stranded DNA fragment lengths in 203 decellularized bronchi was less than 100 bp (Supplemental figure 2).

\section{Protein quantification}

205 Although the difference in total protein concentrations in the native and decellularized

206 bronchi was significant $(\mathrm{p}=0.01)$, the decline in these concentrations remained moderate,

207 to approximately $100 \mathrm{mg} / \mathrm{ml}$ (Fig 2b). The qualitative and semi-quantitative evaluation

208 revealed that collagen I and IV are not affected by the decellularization process. The 
209 fibronectin while decreased, remained detectable in abundant amounts in the

210 decellularized extracellular matrix (Fig 3k-31).

\section{Recellularization assessment}

212 Five different equine bronchi were recellularized with 3 primary ASM cell lines between

213 passages 3 and 7. Recellularizations from 48 hours to 7 days were modest or absent in all

214 cell-tissue combinations tested $(n=19)$. However, between days 14 and 21, the ASM

215 cells were detectable in tissue in 17 out of 19 replicates made during 7 different

216 recellularization trials.

217 At 31 days, ASM cells were observed in all recellularized tissues $(n=20)$. On 4 different

218 recellularization assays, the amount of ASM cells within the scaffold was maximum at 41

219 days. The ASM cells that repopulated the decellularized bronchi were first located on the

220 surface of the basement membrane or in the extracellular smooth muscle matrix (14

221 days). At day 21, ASM cells were present in the smooth muscle layer of the

222 decellularized bronchi and appeared to colonize it preferentially (Fig 4). This was

223 confirmed between 31 and 41 days, with abundant cells in the muscular extracellular

224 matrix and adjacent to the bronchial cartilage. However, some cells remained located

225 above the basement membrane. In two recellularization trials with 3 biological replicates,

226 the recellularization was improved at 41 days by transferring the tissue at day 31 in

227 another well for 10 additional days of culture before harvesting.

228 The immunofluorescent staining confirmed the expression of $\alpha$-SMA by the cells present

229 in zones of abundant recellularization in the smooth muscle matrix (Fig 5). 
230 On scanning electron microscopy analysis, recellularization was identified in most

231 samples on the tissue surface. On some parts of the ECM, where the resistance seamed

232 reduced, the cells penetrated the tissues, as it is shown, Fig 6.

\section{Discussion}

234 In this study, we developed a protocol to recellularize decellularized respiratory bronchi

235 with ASM cells. The results showed a preferential migration and colonization of the

236 muscular extracellular matrix by these cells. This phenomenon is, to the best of our

237 knowledge, reported for the first time in any species. The protocol presented herein will

238 enable the study of the phenotypic changes of ASM cells by an asthmatic ECM.

239 The initial aim of tissue engineering was to develop organs devoid of immunogenic

240 rejections for transplantation [14, 15]. It was then adopted in pharmacological and

241 oncological researches to identify the cellular response to drugs and in diseases [16-18],

242 as the cells' behavior was shown to vary depending on the 3D structures of the substrate

$243[6,19]$. Different organs, including lung tissues, have been decellularized and then 244 recellularized [20-23], mainly with stem cells [24, 25]. Recent studies have recellularized

245 horse lung tissues with equine dermal fibroblasts and canine yolk sac cells [26], and 246 mouse lungs with human and murine fibroblasts [27]. McClure et al. grafted a 247 decellularized skeletal muscle in the gastrocnemius and demonstrated a regeneration of 248 the graft with the presence of cells and neuromuscular junctions [28]. However, none of 249 the studies reported a preferential recellularization for specific cell types when seeded on 250 a heterogenous biological matrix. 
251 To the best of knowledge, the present study is the first to attempt to recellularize airways

252 with primary airway smooth muscle cells. Different combinations of smooth muscle cells

253 lineages and matrices were studied and resulted in the colonization of the bronchial

254 smooth muscle matrix by the ASM cells. This preferential colonization likely involved

255 complex cellular mechanisms including integrin expression, adhesion, migration and

256 proliferation and suggests that the decellularized tissue retained enough of its native

257 qualities to allow this process to occur. Da Palma et al. demonstrated that the fibroblasts

258 recellularizing a decellularized horse lung are expressing the N-cadherin, an adhesion

259 biomarker [26]. This protein may play an important role in the ASM cell migration seen

260 in our study as it has been shown that the migration of the vascular smooth muscle cells is

261 delayed by the inhibition or the down-regulation of the $\mathrm{N}$-cadherin [29, 30]. Moreover,

262 the ECM is known to regulate the migration of the ASM cells [31, 32].

263 The smooth muscle colonization was uneven as some parts of the bronchi contained more

264 ASM cells than other. This unevenness in cell distribution has also been observed during

265 lung recellularization by endothelial cells [33]. Uygun et al. also described variable

266 hepatocytes distribution within hepatic matrix [34]. This may be due to the uneven

267 mechanical properties of the decellularized bronchi as scan electron microscopy results

268 suggest that cells seem to reach the smooth muscle ECM from zones with low tissue

269 resistance.

270 The presence of matrikines in the scaffolds may also have contributed to this preferential

271 cell colonization. Matrikines are peptides produced from the proteolytic degradation of

272 the extracellular matrix [35]. Given the cellular destruction that occurs during

273 decellularization, the release of intracellular proteases may have resulted in the 
274 production of a high concentration of matrikines within the scaffolds. These peptides

275 would affect cell behavior across integrins by stimulating the secretion of certain growth

276 factors. It has been shown that valine-glycine-proline-valine-glycine (VGPVG), a

277 hydrophobic elastin matrikine, stimulates smooth muscle cell proliferation [36]. The

278 tripeptide sequence Arginine-Phenylalanine-Lysine (RFK) derived from

279 thrombospondin-I is also mitogenic to smooth muscle cells through the activation of the

280 transforming growth factor (TGF) $\beta$ [37]. Another peptide, valine-glycine-valine-arginine-

281 proline-glycine (VGVAPG), is chemotactic for fibroblasts [38]. These matrix fragments

282 being mobile and regulating cell behavior, would be potential promoters of myocyte

283 migration observed during recellularization.

284 GAGs are involved in different biological processes including extracellular matrix-cell

285 interaction and activation of various chemokines [39]. They are stained blue on histology

286 using Movat Pentachrome. Interestingly, from the $31^{\text {st }}$ day of recellularization, a blue

287 coloration in the recellularized zones appeared on the Movat Pentachrome histological

288 staining in 4 of 20 bronchi studied. These findings suggest the secretion of GAGs by the

289 ASM cells, which are known to be secretory of these mucopolysaccharides [40].

290 No decellularization method is, to date, able to offer a complete elimination of the 291 cellular material [41]. Thus, four criteria are used to assess the quality of a decellularized

292 matrix: 1) maintenance of matrixial structural protein content, 2) absence of cellular 293 material on histological staining, 3) double stranded DNA should be less than $50 \mathrm{ng} / \mathrm{mg}$

294 tissue and 4) less than $200 \mathrm{bp}$ in length [42, 43]. In agreement with these reports, the 295 bronchial extracellular matrix was histologically free of cellular material with a global 296 maintenance of the bronchial architecture. The DNA concentrations we obtained by 
297 fluorometry and electrophoresis were below the thresholds recommended to avoid in

298 vitro cytocompatibility problems [43]. However, in the context of regenerative medicine,

299 Allman et al. showed that the immunoreactivity of the remnant DNA in a transplanted

300 decellularized matrix could induce graft acceptance through a Th2 type response [44].

301 The decellularization protocol that was implemented herein was previously shown to

302 allow a better preservation of the extracellular matrix than other methods $[11,45]$. The

303 total proteins fluorometric quantification revealed a significant concentration decrease of

304 matrixial proteins in the decellularized bronchi compared to the native ones. This

305 variation was expected as different intracellular proteins are eliminated during this

306 process but the effects on the matrixial proteins seem to depend on the nature of the

307 treated tissue $[46,47]$. It has been shown that some cytoskeletal proteins, including $\alpha$ -

308 SMA and SMMHC, could be detected in decellularized matrices [11, 47, 48] and

309 correspond to the cellular residues observed by electron microscopy [48]. Our results are

310 in agreement with these findings as on confocal microscopy, our decellularized matrices

311 showed staining for $\alpha$-SMA in some smooth muscle matrix areas. Moreover, the

312 decellularized bronchi maintained their general architecture and protein composition

313 almost unchanged in collagen I and IV, elastic fibers and fibronectin based on

314 histological evaluation and semi-quantitative immunohistochemical scoring of native and

315 decellularized bronchi. Those observations were in agreement with previous reports

316 assessing the maintenance of these matrixial proteins [11, 14, 45, 48, 49]. Collagens and

317 fibronectin are important for recellularization. Among other roles, fibronectin allow cell

318 adhesion to the ECM and collagens are needed for their infiltration into it [47]. Laminin 
319 and other matrixial proteins also have cellular adhesion properties and may affect cell

320 behavior and phenotype [50].

321 To conclude, we obtained a decellularized bronchial ECM that was successfully, while

322 incompletely, recellularized with primary mature ASM cells over 41 days of culture. We

323 described a preferential colonization of the smooth muscle ECM by these cells. Other

324 investigations would be necessary to identify the factors and proteins that may be

325 implicated in the ASM cell-specific recellularization observed.

\section{Acknowledgements}

327 The authors thank Dr. Guy Beauchamp for the statistical analysis and the Respiratory

328 Health Network of Quebec (RHN) for the tissue bank creation.

\section{Conflict of interest statement}

330 On behalf of all authors, the corresponding author states that there is no conflict of 331 interest. 


\section{Funding}

333 This project was funded by the Canadian institute of health research (CIHR) (JPL: Grant

334 number PJT-148807) and supported by the faculty of superior and postdoctoral studies

335 (FESP) scholarships (SBH).

\section{Ethical approval}

337 All applicable international, national, and/or institutional guidelines for the care and use

338 of animals were followed. The experimental protocol was approved by the ethical

339 committee of the University of Montreal number Rech-1578.

\section{References}

1. Gibson PG. What do non-eosinophilic asthma and airway remodelling tell us about persistent asthma? Thorax. 2007;62(12):1034-6.

2. Chetta A, Marangio E, Olivieri D. Inhaled steroids and airway remodelling in asthma. Acta Biomed. 2003;74(3):121-5.

3. Cukierman E, Pankov R, Stevens DR, Yamada KM. Taking cell-matrix adhesions to the third dimension. Science. 2001;294(5547):1708-12.

4. Baharvand H, Hashemi SM, Kazemi Ashtiani S, Farrokhi A. Differentiation of human embryonic stem cells into hepatocytes in $2 \mathrm{D}$ and $3 \mathrm{D}$ culture systems in vitro. Int $\mathrm{J}$ Dev Biol. 2006;50(7):645-52.

5. Htwe SS, Harrington H, Knox A, Rose F, Aylott J, Haycock JW, et al. Investigating NF-kappaB signaling in lung fibroblasts in 2D and 3D culture systems. Respir Res [Internet]. 2015 Dec 1 PMC4666055]; 16:[1-9 pp.]. Available from: https://www.ncbi.nlm.nih.gov/pubmed/26619903. 
360 6. Duval K, Grover H, Han LH, Mou Y, Pegoraro AF, Fredberg J, et al. Modeling 361 Physiological Events in 2D vs. 3D Cell Culture. Physiology (Bethesda). 2017;32(4):26636277.

7. Kapalczynska M, Kolenda T, Przybyla W, Zajaczkowska M, Teresiak A, Filas V, et al. 2D and 3D cell cultures - a comparison of different types of cancer cell cultures. Arch Med Sci. 2018;14(4):910-9.

8. Bullone M, Lavoie JP. The equine asthma model of airway remodeling: from a veterinary to a human perspective. Cell Tissue Res [Internet]. 2019 Nov 12. Available from: https://www.ncbi.nlm.nih.gov/pubmed/31713728. asthma-like equine disease, involves airway smooth muscle remodeling. J Allergy Clin Immunol. 2006;118(2):382-8. an equine asthma model. Am J Respir Cell Mol Biol. 2012;47(5):589-96. studies of lung disease and regeneration. Biomaterials. 2014;35(9):2664-79.

12. Vargas A, Peltier A, Dube J, Lefebvre-Lavoie J, Moulin V, Goulet F, et al. Evaluation of contractile phenotype in airway smooth muscle cells isolated from endobronchial biopsy and tissue specimens from horses. Am J Vet Res. 2017;78(3):35970 .

13. Russell HK, Jr. A modification of Movat's pentachrome stain. Arch Pathol. 1972;94(2):187-91. enzymatic treatment. Biomaterials. 2013;34(28):6638-48. 
16. Langhans SA. Three-Dimensional in Vitro Cell Culture Models in Drug p.]. Available from: https://www.ncbi.nlm.nih.gov/pubmed/29410625.

17. Yamada KM, Cukierman E. Modeling tissue morphogenesis and cancer in 3D.

18. Fisher SA, Tam RY, Fokina A, Mahmoodi MM, Distefano MD, Shoichet MS. Photo-immobilized EGF chemical gradients differentially impact breast cancer cell invasion and drug response in defined 3D hydrogels. Biomaterials. 2018;178:751-66. systems and their applications in drug discovery and cell-based biosensors. Assay and drug development technologies. 2014;12(4):207-18.

20. Totonelli G, Maghsoudlou P, Garriboli M, Riegler J, Orlando G, Burns AJ, et al. A rat decellularized small bowel scaffold that preserves villus-crypt architecture for intestinal regeneration. Biomaterials. 2012;33(12):3401-10.

21. Taylor DA, Sampaio LC, Cabello R, Elgalad A, Parikh R, Wood RP, et al. Decellularization of Whole Human Heart Inside a Pressurized Pouch in an Inverted Orientation. J Vis Exp [Internet]. 2018 Nov 26; (141). Available from: https://www.ncbi.nlm.nih.gov/pubmed/30531712. preserved decellularized kidney scaffold using adipose tissue-derived stem cells. J Biomed Mater Res A. 2018;106(3):805-14.

23. Stahl EC, Bonvillain RW, Skillen CD, Burger BL, Hara H, Lee W, et al.

Evaluation of the host immune response to decellularized lung scaffolds derived from alpha-Gal knockout pigs in a non-human primate model. Biomaterials. 2018;187:93-104. culture. PLoS One [Internet]. 2015 PMC4427280]; 10(5):[e0126846 p.]. Available from: https://www.ncbi.nlm.nih.gov/pubmed/25962111. adipose-derived stromal cells. Sci Rep [Internet]. 2017 Aug 16 PMC5559597]; 7(1):[8447 p.]. Available from: https://www.ncbi.nlm.nih.gov/pubmed/28814761. 
26. da Palma RK, Fratini P, Schiavo Matias GS, Cereta AD, Guimaraes LL, Anunciacao ARA, et al. Equine lung decellularization: a potential approach for in vitro modeling the role of the extracellular matrix in asthma. J Tissue Eng. 2018;9:1-11.

Distinct niches within the extracellular matrix dictate fibroblast function in (cell free) 3D lung tissue cultures. Am J Physiol Lung Cell Mol Physiol. 2018;314(5):708-23.

28. McClure MJ, Cohen DJ, Ramey AN, Bivens CB, Mallu S, Isaacs JE, et al.

29. Lyon CA, Koutsouki E, Aguilera CM, Blaschuk OW, George SJ. Inhibition of Ncadherin retards smooth muscle cell migration and intimal thickening via induction of apoptosis. J Vasc Surg. 2010;52(5):1301-9. PMC6147913]; 38(5). Available from: https://www.ncbi.nlm.nih.gov/pubmed/29752344.

31. Parameswaran K, Radford K, Zuo J, Janssen LJ, O'Byrne PM, Cox PG.

Extracellular matrix regulates human airway smooth muscle cell migration. Eur Respir J. 2004;24(4):545-51.

32. Madison JM. Migration of airway smooth muscle cells. Am J Respir Cell Mol Biol. 2003;29(1):8-11.

33. Scarritt ME, Pashos NC, Motherwell JM, Eagle ZR, Burkett BJ, Gregory AN, et al. Re-endothelialization of rat lung scaffolds through passive, gravity-driven seeding of segment-specific pulmonary endothelial cells. J Tissue Eng Regen Med. 2018;12(2):786806. chronic obstructive pulmonary disease. In: Gerbino A, Zummo G, Crescimanno G, editors. Experimental medicine reviews Plumelia Ricerca ed2007. p. 328. 
36. Wachi H, Seyama Y, Yamashita S, Suganami H, Uemura Y, Okamoto K, et al. Stimulation of cell proliferation and autoregulation of elastin expression by elastin peptide VPGVG in cultured chick vascular smooth muscle cells. FEBS Lett. 1995;368(2):215-9.

37. Ribeiro SM, Poczatek M, Schultz-Cherry S, Villain M, Murphy-Ullrich JE. The activation sequence of thrombospondin-1 interacts with the latency-associated peptide to regulate activation of latent transforming growth factor-beta. The Journal of biological chemistry. 1999;274(19):13586-93.

38. Senior RM, Griffin GL, Mecham RP, Wrenn DS, Prasad KU, Urry DW. Val-GlyVal-Ala-Pro-Gly, a repeating peptide in elastin, is chemotactic for fibroblasts and monocytes. J Cell Biol. 1984;99(3):870-4.

39. Papakonstantinou E, Karakiulakis G. The 'sweet' and 'bitter' involvement of glycosaminoglycans in lung diseases: pharmacotherapeutic relevance. Br J Pharmacol. 2009;157(7):1111-27.

40. Nigro J, Wang A, Mukhopadhyay D, Lauer M, Midura RJ, Sackstein R, et al. Regulation of heparan sulfate and chondroitin sulfate glycosaminoglycan biosynthesis by 4-fluoro-glucosamine in murine airway smooth muscle cells. The Journal of biological chemistry. 2009;284(25):16832-9.

41. Gilbert TW, Freund JM, Badylak SF. Quantification of DNA biologic scaffold materials. The journal of surgical research. 2009;1(152):135-9.

42. Gilpin A, Yang Y. Decellularization Strategies for Regenerative Medicine: From Processing Techniques to Applications. Biomed Res Int [Internet]. 2017 PMC5429943]; 2017:[9831534 p.]. Available from: https://www.ncbi.nlm.nih.gov/pubmed/28540307.

43. Crapo PM, Gilbert TW, Badylak SF. An overview of tissue and whole organ decellularization processes. Biomaterials. 2011;32(12):3233-43.

44. Allman AJ, McPherson TB, Badylak SF, Merrill LC, Kallakury B, Sheehan C, et al. Xenogeneic extracellular matrix grafts elicit a TH2-restricted immune response. Transplantation. 2001;71(11):1631-40.

45. Tsuchiya T, Sivarapatna A, Rocco K, Nanashima A, Nagayasu T, Niklason LE. Future prospects for tissue engineered lung transplantation: decellularization and recellularization-based whole lung regeneration. Organogenesis. 2014;10(2):196-207. 
524 46. Grauss RW, Hazekamp MG, Oppenhuizen F, van Munsteren CJ, Gittenberger-de 525 Groot AC, DeRuiter MC. Histological evaluation of decellularised porcine aortic valves: 526 matrix changes due to different decellularisation methods. Eur J Cardiothorac Surg.

$527 \quad 2005 ; 27(4): 566-71$. PMC5340383]; 12(3). Available from: https://www.ncbi.nlm.nih.gov/pubmed/28267813. 


\section{Figures and supplemental Figures legends}

547 Fig 1. Movat Pentachrome staining of a native and a decellularized bronchus at

548 magnification 100. (a) native bronchus, (b) decellularized bronchus. From top to bottom,

549 on each image, the bronchial cartilage, followed by the bronchial smooth muscle

550 surrounded on both sides by the extracellular matrix which, at the level of the lamina

551 propria, is followed by the epithelial cells layer. Collagen is stained in yellow. The black

552 filamentous structures on (a) and on (b) represent the elastic fibers. The cell nuclei are

553 stained in purple, their omnipresence is noted on (a) and their total absence on (b). Scale

554 bars indicate $100 \mu \mathrm{m}$.

555 Fig 2. DNA and total protein concentrations in native and decellularizd bronchi. (a)

556 DNA concentration in $\mathrm{ng} / \mathrm{mg}$ of tissue in 7 native and decellularized bronchi, (b) Total

557 protein concentration in $\mathrm{mg} / \mathrm{ml}$ in 7 native and decellularized bronchi. A significant

558 difference is found for DNA and total protein concentrations between native and

559 decellularized bronchi with respectively, $\mathrm{p}<0.0001$ and $\mathrm{p}=0.01$.

560 Fig 3. Immunohistochemical staining for Collagen I and IV and for fibronectin in

561 native and decellularized bronchi at magnification 100. Native and decellularized

562 bronchi stained for collagen I (a, b, c, d), collagen IV (e, f, g, h) and fibronectin (i, j, k, l).

563 Evaluation of positive staining $(\mathrm{c}, \mathrm{d}, \mathrm{g}, \mathrm{h}, \mathrm{k}, \mathrm{l})$ compared to isotypic controls $(\mathrm{a}, \mathrm{b}, \mathrm{e}, \mathrm{f}, \mathrm{i}$,

564 j). Scale bars indicate $100 \mu \mathrm{m}$.

565 Fig 4. Movat Pentachrome staining at magnification 100 showing the

566 recellularization between day 0 and day 41. The ASM cell nuclei are stained in purple.

567 (a) decellularized bronchi on day 0; (b) recellularized bronchi on day 14 with ASM cells 
568 at the surface and in the collagenous ECM of the smooth muscle; (c) recellularized

569 bronchi on day 21 with ASM cells more organized at the level of the smooth muscle

570 ECM and lined with bronchial cartilage; (d) recellularized bronchi on day 41 with

571 confluent ASM cells in the smooth muscle extracellular matrix and on the surface of the

572 basement membrane. Scale bars indicate $100 \mu \mathrm{m}$.

573 Fig 5. Movat Pentachrome and $\boldsymbol{\alpha}$-SMA immunofluorescence consecutive staining of

574 recellularized bronchial matrix at day 41. (a) and (b): Movat Pentachrome and

575 immunofluorescence staining of the same area in a recellularized bronchus at

576 magnification 100. (c) and (d): Movat Pentachrome and immunofluorescence staining of

577 the same area in a recellularized bronchus at magnification 400 . The cells recellularizing

578 the tissue are expressing the $\alpha$-SMA which is an indication of the smooth muscle nature

579 of the cells colonizing the tissue and their purity. Scale bars are indicating $100 \mu \mathrm{m}$ for (a)

580 and (b) and $25 \mu \mathrm{m}$ for (c) and (d).

581 Fig 6. Scan electron microscopy of decellularized and 41 days recellularized

582 bronchial matrices. (a) decellularized matrix; (b) recellularized matrix with cells

583 appearing on the surface; (c) cross section of recellularized matrix, cells are only on the

584 surface but not inside the tissue; (d) cross section of recellularized matrix, cells are

585 penetrating the tissue. The red arrows are indicating some of the cells. Scale bars are

586 indicating $3 \mu \mathrm{m}$ for (a) and (b) and $30 \mu \mathrm{m}$ for (c) and (d).

587 S1 Fig. Time chart of the recellularizations. $\mathrm{R}$ is indicating recellularization at day 0

588 and $\mathrm{S}$ is referring to sampling on the timeline. 
bioRxiv preprint doi: https://doi.org/10.1101/2019.12.23.878645; this version posted May 13, 2020. The copyright holder for this preprint (which

was not certified by peer review) is the author/funder, who has granted bioRxiv a license to display the preprint in perpetuity. It is made available under aCC-BY-NC-ND 4.0 International license.

589 S2 Fig. DNA electrophoresis for native and decellularized bronchi. The abbreviation

$590 \mathrm{~N} . \mathrm{Br}$ is for native bronchi and D.Br for decellularized ones. 

1 S is

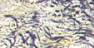
$+4,103 x^{2}$ $7,-\frac{1}{7}, 10$

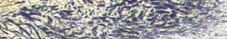
ontsing H hes 
a

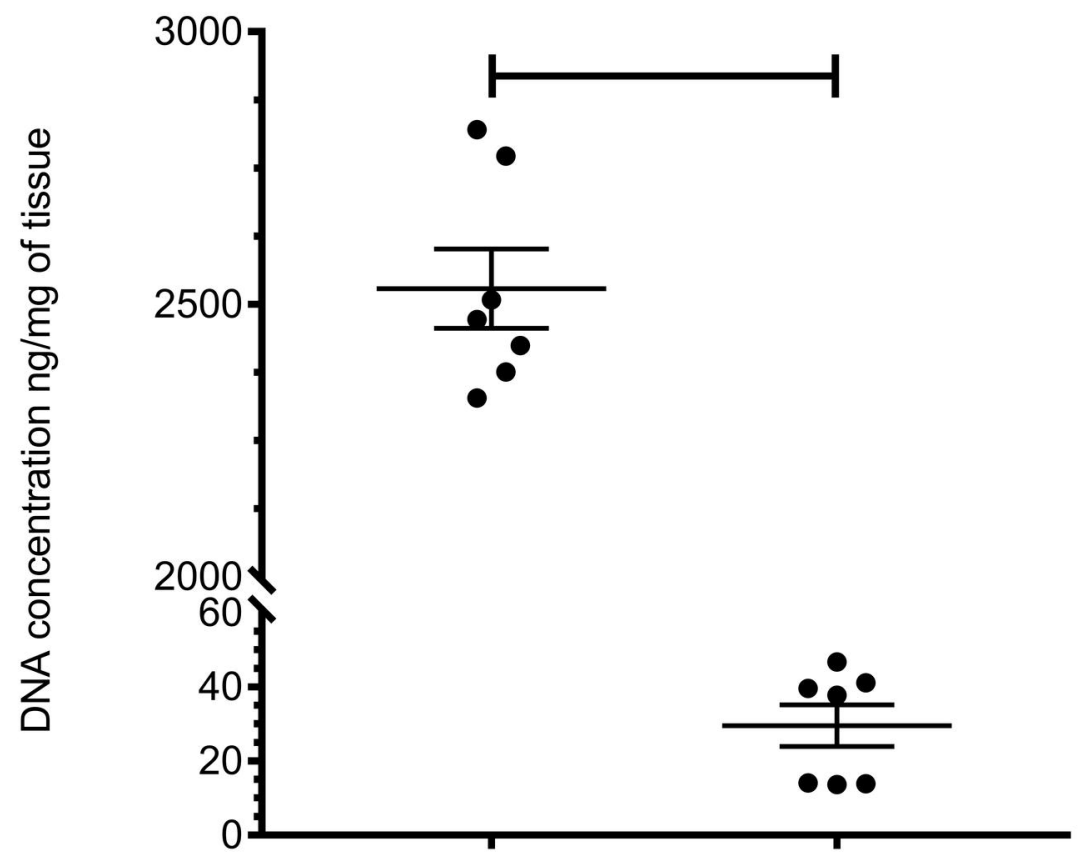

b

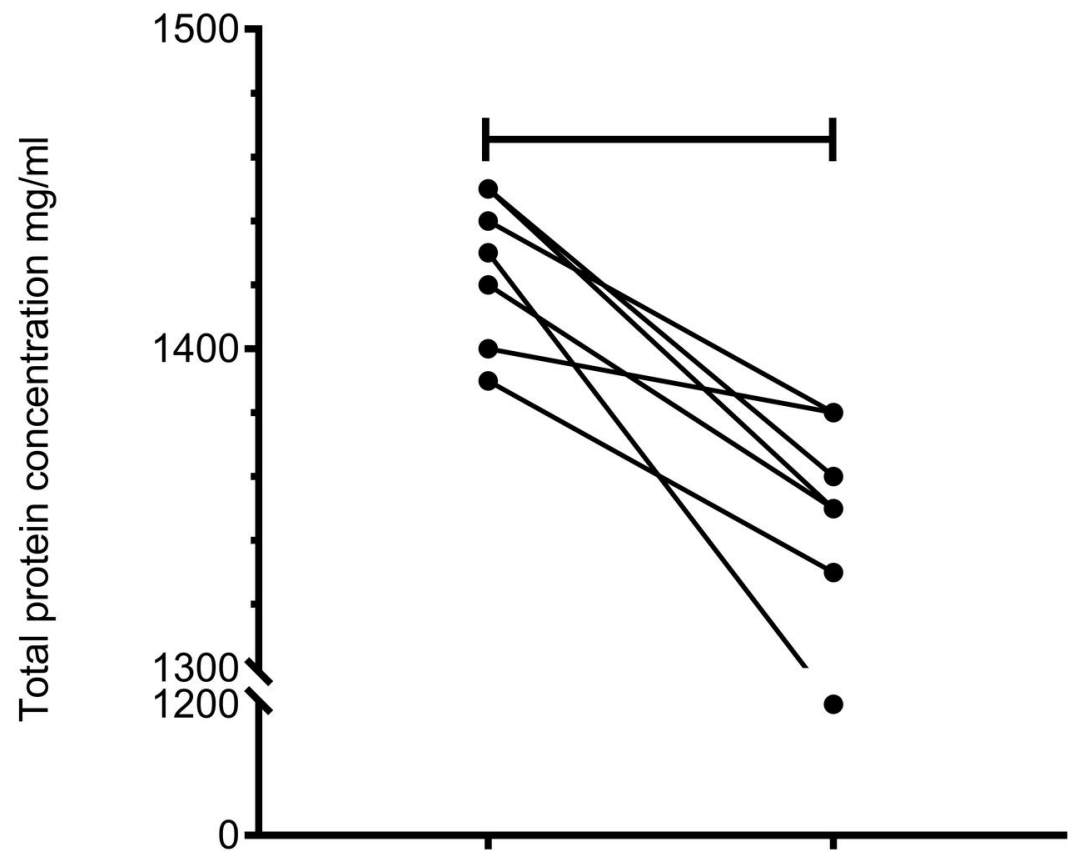

Native bronchi Decellularized bronchi 
a
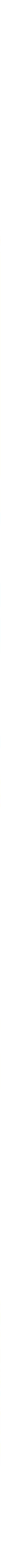


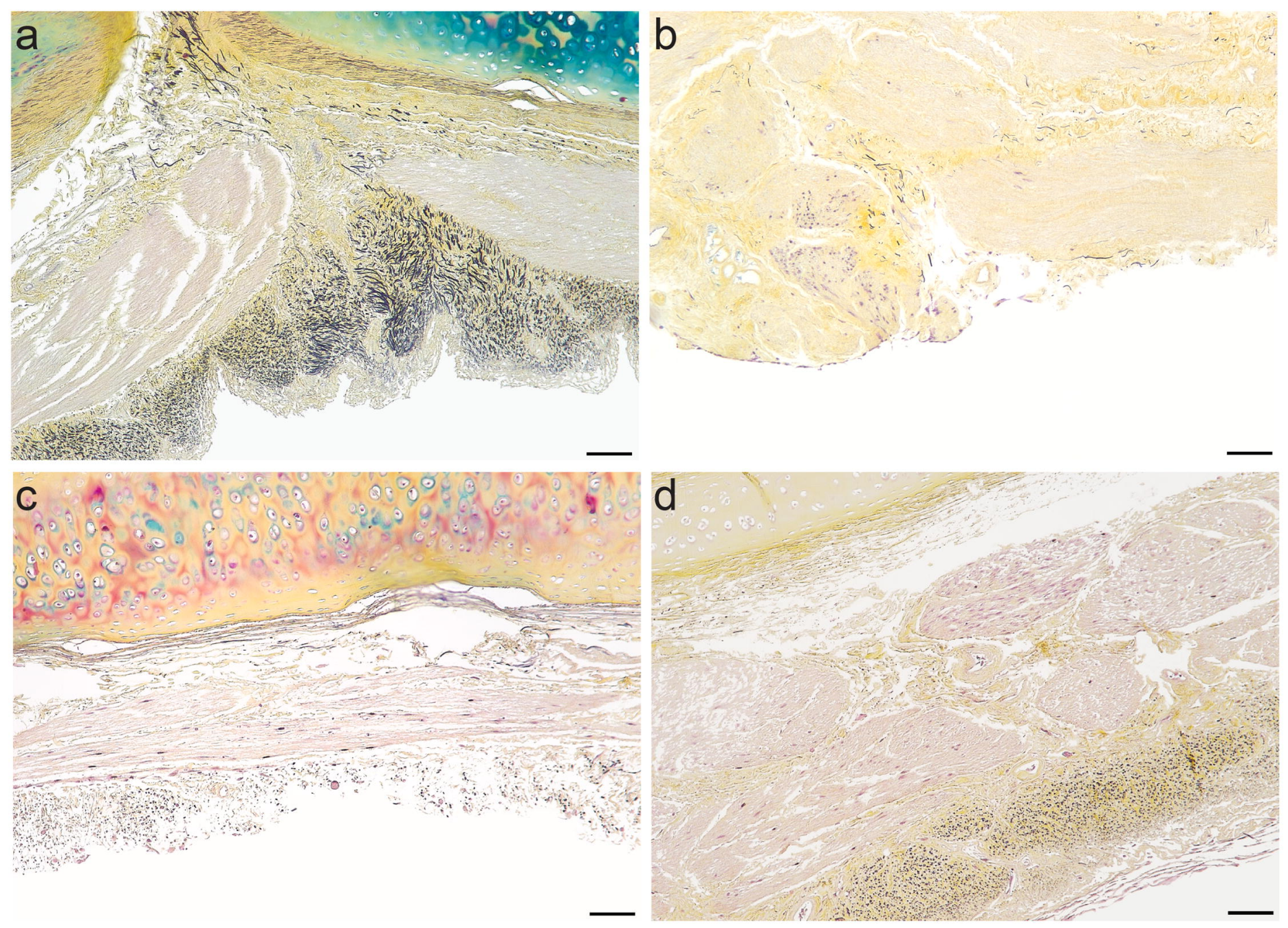




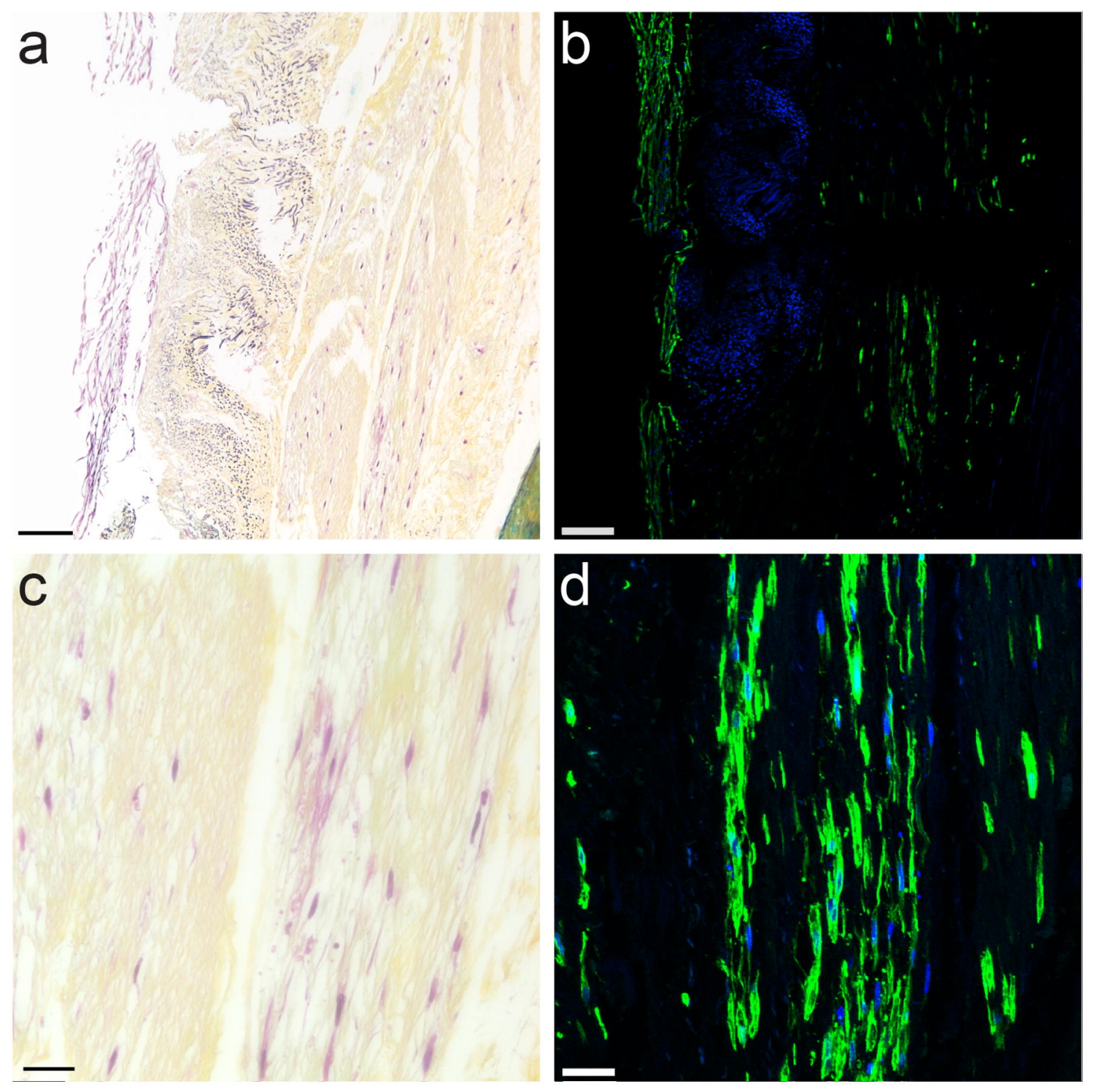




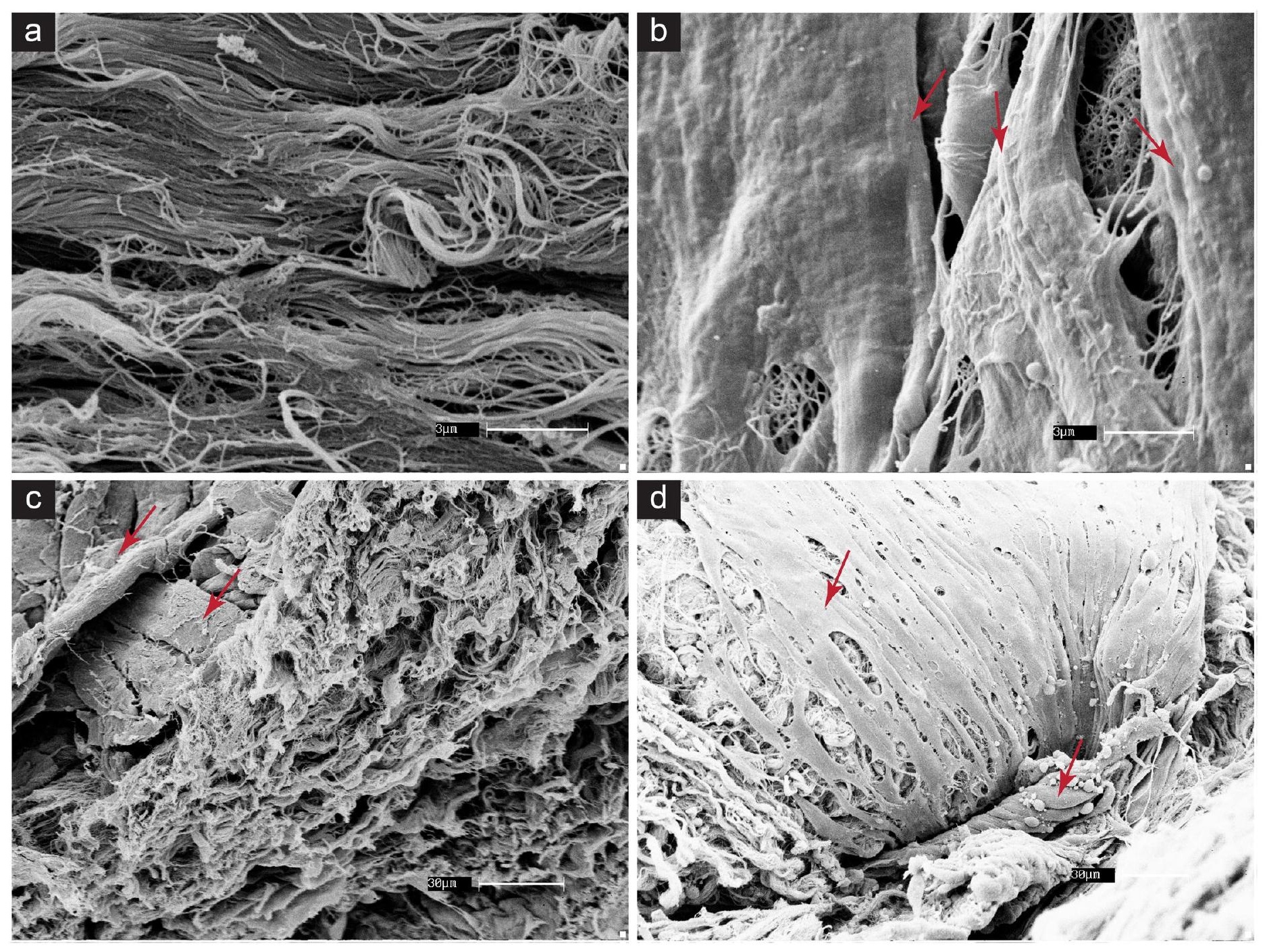

